\title{
Therapeutic effects of mesenchymal stem cells- conditioned medium derived from suspension cultivation or Silymarin on liver failure mice
}

Fatemeh Amiri ( $\square$ amirif2012@gmail.com )

Hamadan University of Medical Sciences https://orcid.org/0000-0002-9976-4734

Sedigheh Molaei

Qom University of Medical Sciences

Rasoul Salimi

Hamadan University of Medical Sciences

Shirin Ferdowsi

Iranian Blood Transfusion Organization

Marzie Bahadori

Iranian Blood Transfusion Organization

\section{Research Article}

Keywords: Conditioned medium, Silymarin, Liver failure, RIPK1 protein, MIRN122

Posted Date: January 31st, 2022

DOI: https://doi.org/10.21203/rs.3.rs-1284048/v1

License: (c) (1) This work is licensed under a Creative Commons Attribution 4.0 International License.

Read Full License 


\section{Abstract}

Background: Common treatments of liver disease failed to meet all the needs in this important medical field. It results in an urgent need for proper some new adjuvant therapies. Mesenchymal stem cells (MSCs) and their derivatives are promising tools in this regard. We aimed to compare the Silymarin, as traditional treatment with mesenchymal stem cell conditioned medium (MSC-CM), as a novel strategy, both with therapeutic potentialities in term of liver failure (LF) treatment.

Methods and Results: Mice models with liver failure were induced with $\mathrm{CCl}_{4}$ and were treated in the groups as follows: normal mice receiving DMEM-LG medium as control, LF-mice receiving DMEM-LG medium as sham, LF-mice receiving Silymarin as LF-SM, and LF-mice receiving Sph-MSC-CM as LF-MSC$\mathrm{CM}$. Biochemical, histopathological, molecular and protein level parameters were evaluated using blood and liver samples. Liver enzymes, MicroRNA-122 values as well as necrotic score were significantly lower in the LF-SM and LF-MSC-CM groups compared to sham. LF-SM showed significantly higher level of total antioxidant capacity and malondialdehyde than that of LF-MSC-CM groups. Sph-MSC-CM not only induced more down-regulated expression of fibrinogen-like protein 1 and receptor interacting protein kinases 1 but also led to higher expression level of keratinocyte growth factor. LF-MSC-CM showed less mortality rate compared to other groups.

Conclusions: Hepato-protective potentialities of Sph-MSC-CM are comparable to those of Silymarin. More Inhibition of necroptosis/ necrosis and inflammation might result in rapid liver repair in case of MSC-CM administration.

\section{Introduction}

Liver diseases are kind of condition that have frequent rate of related mortality [1-3]. Excessive alcohol intake, obesity, viral hepatitis infections and modern lifestyle are the most important risk factors being leading to make a new epidemic wave of liver diseases [4, 5].

Treatment options for common liver diseases such as fatty liver and acute liver failure (ALF) [5] [6] are highly problematic. Liver transplantation is a one of the most common treatment of these sever diseases; however, it is associated with important challenges such as liver donor shortage, risk of immune rejection and side effects of immunosuppressive drugs $[7,8]$.

Botanical medicine has been used traditionally as herbal therapy worldwide for the prevention and treatment of liver disease [9]. In traditional medicine, Silybum marianum is one of the plants which is widely prescribed for the treatment of liver disorders [10-12]. Silybum marianum, also known as milk thistle, contains a unique flavonoid complex called Silymarin which contains Silydianin, Silybin and Silychrisin, and is thought to have liver protective properties $[13,14]$. Both in vitro and in vivo researches suggest that Silymarin has protective properties for liver cells against toxins [11, 13-15]. Feher et al (2012) mentiond Silymarin as an adjuvant therapy for hepatocellular carcinoma due to its antiinflammatory effects [16]. 
In recent years, researchers' focus shifted from traditional medicine to mesenchymal stem cells (MSCs)based strategies and novel cell-free strategies in term of clinical treatment $[17,18]$. However, cell-free strategies with several advantages over cell-based strategies shows a promising future in the field of liver disorders $[19,20]$. In this regard, the existing evidence suggests that the MSCs conditioned medium containing secreted soluble molecules, extracellular proteins, exosomes/ microvesicles etc. mediate their principal therapeutic properties through paracrine effects instead of transdifferentiation mechanism in order to substitute injured cells [21-23]. Bioactive molecules of MSC-conditioned medium (MSC-CM) such as growth factors, angiogenic factors and proteases [22-25] involve in either physiological process regulation or pathological condition management $[23,26]$ especially in acute liver diseases [25].

According to available published study and our experience, we aimed to compare the Silymarin, as traditional treatment with mesenchymal stem cell conditioned medium (MSC-CM), as a novel strategy, both with therapeutic potentialities in term of liver failure (LF) treatment.

\section{Materials And Methods \\ Herbal extract}

Livergol, generic name and brand of Silymarin (Goldaru, Iran) was purchased from a drugstore in the form of $140 \mathrm{mg}$-tablet. The tablets were dissolved in normal saline.

\section{MSCs culture}

In order to prepare MSC-CM, human umbilical-derived MSCs were isolated and characterized as described previously [27]. All protocols were conducted after filling out the consent forms by parents. Next, the isolated MSCs were subjected to usual monolayer adherent or non-adherent spheroid-form cultivation as described previously [27]. Poly-HEMA polymer was used to inhibit MSCs attachment on the surface of cell culture plates.

\section{Preparations of MSCs conditioned medium and their characterization}

MSC-CM was prepared as follows: Either MSCs under adherent condition or those under non- adherent suspension condition which resulted in spheroid colony were cultured in serum-free medium for 48 hours. The supernatants were aspirated gently, filtered through $0.22 \mu \mathrm{m}$-filter and then, transferred to Vivaspin ${ }^{\circledR}$ 500 centrifugal filter units (Sartorius, Germany). Finally, two kinds of CM, MSC-CM and spheroid-MSC-CM (Sph-MSC-CM) were centrifuged, $4000 \mathrm{~g}$ for 30 minutes at $4^{\circ} \mathrm{C}$. Bicinchoninic acid assay kit (Kiazist Life Sciences, Iran) was utilized for protein level determination in MSC-CM and Sph-MSC-CM. The concentration of vascular endothelial growth factor (VEGF), hepatocyte growth factor (HGF) and 
fibroblast growth factor (FGF) were measured using ELISA method (R\&D Systems, USA) according to the manufacturer's protocol.

\section{Animal study}

In this experimental study, 8-week-old Naval Medical Research Institute (NMRI) mice weighting 25 \pm 2 gram were purchased from Pasteur institute of Iran (Tehran, Iran). The mice were raised in our colony and kept in standard condition in term of light, food, and water accessibilities. Food was withheld 12-14 hours before operation or death. In all experiments, the regulations of local authorities for handling laboratory animals and the National Institutes of Health guide for the care and use of laboratory animals (NIH Publications No. 8023, revised 1978) were considered. This study was confirmed in ethic committee of Hamadan university of medical sciences (Code No: IR.UMSHA.REC.1398.1077).

\section{Induction of liver failure in animals}

Carbon tetrachloride, $\mathrm{CCl}_{4}$, (Merck, Germany) was used to produce mice models of experimental liver failure as established in our previous studies $[28,29]$ with some modifications in time period. $1.5 \mathrm{ml} / \mathrm{kg}$ $\mathrm{CCl}_{4}$ dissolved in olive oil was determined as the appropriate dose for liver failure induction and administered intraperitoneally to the mice. The induction of liver failure was confirmed after 12 hours with biochemical, histopathological and molecular methods.

\section{Different treatments of animal groups and study design}

In order to evaluate the hepatoprotective effects of Sph-MSC-CM or Silymarin, the mice were divided into four groups, each containing 10 mice as follow: normal mice receiving DMEM-LG medium as control, LFmice receiving DMEM-LG medium as sham, LF-mice receiving Silymarin as LF-SM and LF-mice receiving Sph-MSC-CM as LF-MSC-CM. The Silymarin optimized injected dose and Sph-MSC-CM adjusted protein concentration were determined. Their toxicity on mice fibroblast cell line and IC50 was determined using standard protocol. Then each group received 250 microliters of the medium or Sph-MSC-CM (adjusted protein concentration: $200 \mu \mathrm{g} / \mathrm{kg}$ ) or Silymarin (adjusted concentration: $100 \mathrm{mg} / \mathrm{kg}$ ) at the same method according to the Institutional Animal Care and Use Committee (IACUC) recommendations intraperitoneally. All treatments were performed 12 hours after the induction of liver failure with $1.5 \mathrm{ml} / \mathrm{kg}$ of dissolved $\mathrm{CCl}_{4}$ in olive oil. Subjected animals received the treatments daily for three consecutive days. Blood samples and liver tissues were collected 12 and 72 hours post treatments.

\section{Evaluation of Liver enzymes}


Blood samples were collected by cardiac puncture technique under deep anesthesia with ketamine (150 $\mathrm{mg} / \mathrm{kg}$ ) and xylazine (15 mg/kg) cocktail (Alfasan, Netherland). Aspartate aminotransferase (AST) and alanine aminotransferase (ALT) were measured. Commercial kits (Parsazmoon, Iran) and automatic analyzer system (BT 3000 PL/S, Italy) were used. Argininosuccinate synthetase (ASS), as sensitive biomarker of liver injury, level was also detected using specific ELISA kit (Mybiosource, Canada).

\section{Preparation of liver tissue homogenates}

The liver tissue was minced to small pieces $(0.5 \mathrm{~g})$ and rinsed with cold phosphate buffer saline. The tissues were primed with lysis buffer (Sigma, USA) and sonicated with an ultrasonic homogenizer (BioLogics, USA). The resulting suspensions were centrifuged at $10000 \mathrm{~g}$ for 5 minutes. Then, their protein levels were determined (Kiazist Life Sciences, Iran). Real-time PCR, western blot analysis and oxidative stress assays were performed on these harvested homogenates immediately or after aliquoting and storing at $\leq-20^{\circ} \mathrm{C}$.

\section{Real-time PCR}

To confirm molecular level expression of intended genes and biomarkers including MicroRNA122 , glutathione peroxidase $(G S H-P X)$, superoxide dismutase $(S O D)$, receptor interacting protein kinases 1 (RIPK1), fibrinogen-like protein 1 (FGL-1) and keratinocyte growth factor (KGF), real-time PCR was performed on liver tissue homogenates. Total RNA was extracted (Kiazist Life Sciences, Iran). The procedure was performed according to the suggested protocols by kit manufacturer. Then, $500 \mathrm{ng} / \mathrm{ml}$ of total RNA was subjected to cDNA synthesis (BIONEER, South Korea). qRT-PCR was performed using SYBR green master mix (Takara, Japan) and designed specific primers (Table 1S). The LightCycler® 96 System (Roche, Bavaria, Germany) was utilized to perform reactions in triplicate. Fold change of gene of interest expression to $\beta$-actin expression was calculated after melting cure analysis using $2^{-\Delta \Delta C t}$ method.

\section{Evaluation of oxidative stress status}

Colorimetric method was applied to determine the level of oxidative stress in liver tissue homogenates. Malondialdehyde (MDA) as a lipid peroxidation marker and total antioxidant capacity (TAC) level was assayed by specific biochemical analysis kits (ZellBio, Germany). GSH-Px and SOD activities were also determined with commercially available kits (ZellBio, Germany) according to the manufacturer's protocols.

\section{Western blot analysis}

Translational level of RIPK1, FGL-1 and KGF were detected in liver tissue homogenates. First, $12 \%$ SDSpolyacrylamide gel was prepared and sample proteins were separated according to their molecular 
weight by electrophoresis. Then, Semi-dry blotter (Bio-Rad, USA) was used for blotting the proteins onto the PVDF membrane (Roche, Germany). The membranes were incubated with primary antibodies including anti-FGL-1 (Abcam, UK), anti-KGF (Abcam, UK), anti-RIPK1 (LifeSpan BioScience, USA) and anti$\beta$-actin (Sigma, USA) after setting up the time, temperature and proper dilution separately. Afterwards, secondary antibody (Abcam, UK) was used on the surface of the membrane. ECL substrate for horseradish peroxidase (Abcam, UK) was added to the membranes, and imaging was performed using gel doc imager (Bio-Rad, USA). The density of protein bands was semi-quantified using ImageLab software.

\section{Histopathological assay}

To detect tissue necrosis and inflammatory cells infiltration, liver tissue sections were analyzed histopathologically. The animals were dissected and their livers were separated and fixed with formalin. After preparation of liver sections, they were stained with hematoxylin and eosin ( $\mathrm{H} \& \mathrm{E})$ to determine the necrosis/ inflammation scores as described in our previous study [28] with some modifications. Necrosis and inflammation level were quantified by estimating the intralobular necrotic bridges, necrotic cells and inflammatory cell numbers under high-power field (HPF) at least in 30 light microscope fields.

\section{Survival assay}

The survival rate of animal groups was analyzed after treatments within two weeks. The mice $(n=10)$ were observed every day in terms of their survival rate.

\section{Statistical analysis}

The quantitative values were given as mean \pm SD. SPSS version 21 software (SPSS Inc., Chicago, IL, USA) and One-way ANOVA test was utilized to data analysis statistically. $p<.05$ was considered as statistically significant. The survival rate was analyzed by Log Rank (Mantel-Cox) test and presented via KaplanMeier plot. ALL experiments were done in triplicate.

\section{Results}

\section{Sph- MSC-CM contains more hepatoprotective growth factors than MSC-CM}

After harvesting MSCs conditioned medium from their cultivation under monolayer adherent (MSC-CM) or spheroid non-adherent (Sph-MSC-CM) conditions, the concentrations of VEGF, HGF and FGF were measured in both mentioned CMs. Protein expression levels of these growth factors were up regulated using suspension cultivation (Fig 1S). As shown in Figure 1S, Sph-MSC-CM contains much more amount 
of these growth factors especially VEGF ( $p<0.001)$. Base on this finding, Sph-MSC-CM was used for the next step of the study.

\section{$1.5 \mathrm{mg} / \mathrm{kg} \mathrm{CCl}_{4}$ induces liver failure in mice}

12 hours after the injection of $1.5 \mathrm{mg} / \mathrm{kg} \mathrm{CCl}_{4}$, the induction of LF was assayed with different laboratory methods. Increased AST and ALT levels were confirmed in $\mathrm{CCL}_{4}$-treated mice in comparison with controls that do not receive any $\mathrm{CCL}_{4}$ by biochemical assay $(p<0.001)$ (Fig $2 \mathrm{Sa}$ ). The results of ELISA were indicated that ASS, as early sensitive liver injury biomarker, concentration was very high in LF-induced mice $(p<0.001)$ (Fig 2Sb). More expression of MicroRNA-122, as a marker of liver injury $(p<0.001)$ was detected using real-time PCR in $\mathrm{CCL}_{4}$-treated group (Fig 2Sc). According to the results of both biochemical and molecular assays, $1.5 \mathrm{mg} / \mathrm{kg}$ of $\mathrm{CCl}_{4}$ administration led to sever liver injury. For more confirmation, liver sections of both the $\mathrm{CCl}_{4}$-treated mice and control groups were evaluated after $\mathrm{H} \& \mathrm{E}$ staining (Fig 2Sd). Extensive intralobular necrotic bridges and multifocal diffusion lobular necrosis were detected in the sections that were accompanied by increasing number of necrotic and inflammatory cells in the portal area or around the necrotic hepatic cells (Fig 2Sd).

\section{Administration of Silymarin or Sph-MSC-CM decreases both the liver enzymes and mir-122 values in LF-mice}

After $\mathrm{CCl}_{4}$ injection, animals were treated by Silymarin (LF-SM) or Sph-MSC-CM (LF-MSC-CM) as experimental groups along with the control groups. The biochemical assays indicated the alterations of both the ALT and AST levels in the serum as well as ASS levels in the experimental groups (Fig $1 \mathrm{a}-\mathrm{C}$ ). 12 hours after treatment, a decrease in the liver enzyme levels were detectable in LF-SM and LF-MSC-CM compared with sham especially in terms of ASS $(p<0.05)$. In the next 72 hours, AST, ALT and ASS were significantly lower in the LF-SM and LF-MSC-CM groups compared with sham group $(p<0.001)$ (Fig $1 \mathrm{a}, \mathrm{b}$, c). As shown in Fig 1d, molecular assay results and decreased level of MicroRNA-122 were consistent with biochemical assay results and revealed the reduction of liver failure in both the LF-SM and LF-MSCCM mice compared to sham $(p<0.05, p<0.001)$ (Fig 1d).

\section{Treatment with Silymarin or Sph-MSC-CM not only up regulates SOD, GSH-PX, FGL-1 and KGFbut also alleviates RIPK1 gene expression}

In order to determine the mechanism underlying Silymarin or Sph-MSC-CM therapeutic effects, transcriptional expression levels of some genes including SOD, GSH-PX, RIPK1, FGL-1 and KGF were evaluated through real-time PCR. The expression levels of SOD, GSH-PX (anti oxidative stress enzymes) 
along with FGL-1 and KGF (regenerative and repairing markers) increased in LF-SM and LF-MSC-CM in comparison with sham $(p<0.01, p<0.001)$ (Fig 2a). The findings indicated that GSH-Px gene expression in liver tissue of LF-SM was a little more than that of LF-MSC-CM $(p<0.05)$. However, the expression of FGL1 enhanced in LF-MSC-CM much more than LF-SM $(p<0.01$ ) (Fig 2a). On the other hand, the administration of Silymarin or Sph-MSC-CM led to a decrease in the level of RIPK1 gene expression (necroptosis/ necrosis marker) $(p<0.01, p<0.001)$ (Fig 2b). However, much less RIPK1expression was detectable in LF-MSC-CM mice ( $p<0.05)$ (Fig $2 b)$.

\section{Injection of Silymarine enhances defense mechanisms against oxidative stress a little more than Sph-MSC-CM.}

For more investigation, the level of MDA and TAC as well as SOD and GSH-Px activities were assayed in the liver homogenates of animal models through specific laboratory methods. Both treatments with Silymarin and Sph-MSC-CM resulted in the improvement of antioxidant capacity compared with no treatment condition $(p<0.01, p<0.001)$. As shown in Fig 3a, more increased TAC level was determined in LF-SM compared with LF-MSC-CM $(p<0.01)$. According to the data in Fig $3 b$, the amount of main lipid peroxidation marker, MDA, was higher in sham against treated animal $(p<0.001)$. MDA content of LFMSC-CM liver homogenates was more than that of LF-SM $(p<0.05)$. Similar to the molecular results for $S O D$ gene expression, SOD enzyme activity was higher $(p<0.001)$ in both experimental groups compared with sham. But its level was not significantly different in both LF-SM and LF-MSC-CM groups (Fig 3c). No significant difference was observed between LF-SM and LF-MSC-CM in terms of GSH-Px activity (Fig 3d). Altogether, Silymarine could reinforce relative antioxidant effects against harsh conditions in LF-SM.

\section{LF-MSC-CM group shows more liver regeneration and less necroptosis/ necrosis protein marker levels}

Using molecular assay for the quantification of mRNA amounts of RIPK1, FGL-1 and KGF, western blot analysis was also performed to detect their translational expression level (Fig 4a). Semi-quantification of protein bands density results showed increasing level of FGL-1 and KGF either in LF-SM or LF-MSC-CM group ( $p<0.01, p<0.001$ ) (Fig 4b). Interestingly, FGL-1 protein was expressed at high measurable level following the injection of Sph-MSC-CM ( $p<0.05)$ (Fig 4b). On the other hand, RIPK1 protein was downregulated in Silymarin and Sph-MSC-CM receiving mice $(p<0.01, p<0.001)$. In consistent with molecular assay results, western blot results also showed much more decreasing protein level of RIPK1 for LF-MSCCM groups compared with LF-SM ( $<<0.05)$ (Fig 4b).

\section{Sph-MSC-CM induces relatively more liver re-construction and less liver inflammation}


The histopathological analysis was performed to evaluate liver repairing in prepared liver section. There were no intralobular necrotic bridges and diffuse necrosis zones, as sever necrosis score, in the liver sections of both Silymarin and Sph-MSC-CM-treated animals (Fig 5a). However, either multiple intralobular necrotic bridges and diffuse necrosis zones or more inflammatory and necrotic cells were detectable in sham (Fig 5a). Moreover, decreased inflammatory and necrotic cells were also observed in histopathological examination of liver sections of LF-SM and LF-MSC-CM ( $<<0.001)$ (Fig 5b) through estimating the numbers of intralobular necrotic bridge, necrotic cells and inflammatory cells. Interestingly, LF-MSC-CM group with less numbers of inflammatory and necrotic cells $(p<0.01)$ might be interpreted as a quick start recovery (Fig $5 b$ ). These data even more confirmed the data gathered from other laboratory techniques. Therefore, the administration of these mentioned treatments not only inhibited necrosis progression in liver but also repaired the damaged tissue with the quick inflammation control compared to sham.

\section{LF-MSC-CM shows higher survival rate}

The survival rate of different animal groups was estimated during two weeks. More mortality rate was seen in the sham group. Sph-MSC-CM-receiving mice, LF-MSC-CM, showed more mean of survival time compared to the sham group and even LF-SM group (Fig 5c).

\section{Discussion}

Applying Silymarin to treat hepatic disease has been reported in several studies in the field of herbal medicine $[11,13,15,16]$. On the other hand, administration of MSCs and their derivatives are attractive cell-base or cell-free therapy in this regard [20,22, 23, 26]. Interestingly, application of umbilical cord MSCs or isolated MSCs from other birth-associated tissues instead of adult tissues offers the opportunity of increasing curative abilities of these valuable cells [30]. In current study, we used Silymarin or conditioned media of umbilical cord MSCs for treatment of liver failure-induced mice. $\mathrm{CCl}_{4}$ was used for the production of reactive oxygen species in order to induce liver failure [28-30].

For preparation of Sph-MSC-CM, umbilical cord-MSCs were cultivated in suspension condition in order to create spheroid colonies [27]. Employment of this strategy improved the ability of subjected MSCs in VEGF, HGF and FGF secretion. MSCs culturing in non-adherent non-monolayer condition results in either enhanced secretory effects or expanded differentiation potentialities of these cells [27, 31].

Our findings indicated that both Silymarin and Sph-MSC-CM ameliorated liver injury and reduced AST, ALT, ASS and MicroRNA-122 levels after intraperitoneal injection to LF mice models. Measurement levels of ASS and MicroRNA-122 along with AST, ALT give a chance to faster confirmation of LF or therapy efficiency. ASS levels increase more rapidly than ALT and AST and make it a reliable sensitive biomarker for the detection, progression and monitoring liver injury [32]. MicroRNA-122 is expressed in liver tissue more than other organs and play important roles in the liver injury and repair [33, 34]. Consistent with our 
results, Hermenean et al (2015) reported the decreasing effects of Silybum Marianum seed oil extract on the activities of liver enzymes such as ALT, AST and gamma glutamyl transferase (GGT) [35]. They pretreated the mice with this herbal oil extract orally at the dose of $10 \mathrm{~g} / \mathrm{kg}$ during a time period of 21 days before the induction of liver failure with $\mathrm{CCl}_{4}[35]$.

In current study, a dose of $100 \mathrm{mg} / \mathrm{kg}$ Silymarin using IP route was administrated for 3 days. In another study, decreased level of ALT and AST was reported 24 hours after the administration of MSC-CM which was prepared by other methods [36].

These treatments caused to reduce the destructive effects of $\mathrm{CCl}_{4}$ on liver tissue such as intralobular necrotic bridges or inflammatory and necrotic cells. More inhibition of necroptosis/ necrosis and faster liver regeneration following the injection of Sph-MSC-CM were also confirmed by molecular and western blot assays. The transcriptional and translational expression levels of lower receptor interacting protein kinases 1 (RIPK1), higher fibrinogen-like protein 1 (FGL-1) and keratinocyte growth factor (KGF) in LFMSC-CM group treated with Sph-MSC-CM might explain the reasons of fast injury inhibition followed by rapid liver regeneration. Apoptosis, autophagy, necrosis and necroptosis that might be mediated by RIPK1 are different models of liver cell death under physiologic and pathologic conditions [37]. Among them, necroptosis that occur in RIPK1-dependent or/and RIPK1-independent pathways and its proper regulation/ prevention play an important role in liver regeneration and hemostasis [38]. Necroptosis, also known as apoptosis and necrosis cross-talk, is kind of a programmed cell death that occurs on apoptosis back up and necrosis morphology [38].

FGL1, also known as hepassocin, increases hepatocyte proliferation by enhancing $3 \mathrm{H}$ thymidine uptake and leads to liver regeneration [39]. Moreover, MSC-CM cytokine contents such as IL-6 could up regulate the expression of FGL-1 and result in rapid liver restoration [40]. MSCs and their conditioned medium stimulate KGF, a fibroblast growth factor family member, activation and positively influence the fate of liver diseases [41, 42]. Xagorari et al (2013) confirmed both the anti-apoptotic and regenerative effects of MSC-CM on hepatocytes in vitro and in vivo by assessing the expression levels of annexin V/PI and FGL$1[40]$.

In order to make more understanding of the possible effects of Silymarin and Sph-MSC-CM on LF-mice, oxidative stress status was analyzed by biochemical measurement of TAC and MDA. Furthermore, molecular assessment of SOD and GSH-Px as well as the recently-mentioned activity evaluations were performed. Despite the ability of Sph-MSC-CM to address harsh $\mathrm{CCl}_{4}$ induced oxidative stress, Silymarin administration proved to be much more effective. It not only induced an increase in TAC level, but also led to more reduction in MDA level. Silybin, the most common composition of Silymarin, is known for its different therapeutic properties. Not only it has antioxidant, anti-inflammatory and antiviral effects, but also it involves in mitochondrial function regulation $[13,14,43,44]$. In the other word, another composition of Silybin, Taxifolin, acts as strong antioxidant $[43,44]$. 
In another study, decreased MDA level and increased enzyme activities of SOD, GSH-Px and catalase were detected following oral administration of Silymarin [35]. Pouyandeh Ravan et al (2019) showed the capacity of MSC-CM either to return the TAC content of hepatocytes or to control the total oxidative status and MDA production in liver fibrosis [30].

Moreover, in current study, a relatively higher survival rate was observed in Sph-MSC-CM-receiving mice. Lotfinia et al (2016), harvested the CM from embryonic stem cell-derived MSCs and assayed its therapeutic effects on thioacetamide-induced LF mice (400 $\mu \mathrm{L}-\mathrm{IP}$ injection). Their results indicated that $\mathrm{CM}$ accelerates liver restoration through the induction of hepatocyte proliferation due to its immunomodulatory properties. However, it had no effect on mice survival rate [45]. On the other hand, they implied that the injection of recombinant VEGF led to more survival rate in the animal models [49]. In current study, Sph-MSC-CM derived from spheroid-form MSCs had approximately 4 times more VEGF content than that of MSC-CM derived from usual monolayer MSCs.

Many research studies have been conducted to evaluate the efficiency of herbal extracts or MSCs conditioned medium in liver failure treatments $[10,11,13,20,23,25,26]$; however, few studies are available in terms of examining the effects of these two fields in the same experiment in order to make better prospective of adjuvant therapies.

In a study, the anti-fibrotic effect of MSC-CM on hepatic stellate cells was confirmed both in vitro and in vivo through the inhibition of TGF $\beta$ signaling pathway [46]. They administered thioacetamide or $\mathrm{CCl}_{4}$ to induce liver fibrosis in mice. After 24 hours, the animals were subjected to intraperitoneal injection of MSC-CM, 500 $\mathrm{gg} / \mathrm{kg}$ [46]. We evaluate the therapeutic potentialities of MSC-CM 12 hours after administration of $200 \mu \mathrm{g} / \mathrm{kg}$ of Sph-MSC-CM. Huang et al (2016) infused $200 \mu \mathrm{l}$ of MSC-CM or intact MSCs into the tail vein of both fulminant hepatic failure and liver fibrosis mice. Their results indicated that the anti-inflammatory property of MSCs and their CM led to a partial restoration of liver tissue while it had no effect on the animal survival rate [47]. They concluded that MSC-CM could be more efficient to be addressed for liver fibrosis complications than liver fulminant failure [47]. MSC-CM has made a breakthrough in regenerative medicine due to its safety, effectiveness, ease of delivery to the patients and no ethical complications in comparison with cell-based therapies [18-20].

Silymarin usage for the treatment of liver diseases was investigated in different studies $[11,14,43]$. Koçarslan (2016) evaluated hepatpprotetive potentialities of Silymarin on rats with liver fibrosis using biochemical and histopathological methods. They used IP injection of Silymarin at the dose of 200 $\mathrm{mg} / \mathrm{kg}$ [44].Consistent with our findings; this study also reported an enhancement in TAC level and reduction in liver necrosis and inflammation. In another study, $50 \mathrm{mg}$ of Silymarin flavonoids was administrated to mice via IP or oral route in a 7-day-period of time [48]. Bilirubin-mediated antioxidant effects of this herbal extract was emphasized in comparison to control [48].

\section{Conclusion}


In conclusion, both Silymarin and Sph-MSC-CM have hepatoprotective effects. However, the latter showed rapid inhibition of liver necroptosis/ necrosis and inflammation. It indicates more promising results according to the survival rates. Strong hepatoprotective characteristics of Sph-MSC-CM could be due to the high content of useful growth factors including VEGF, HGF and FGF as well as anti-inflammatory, antiapoptotic and anti-necrotic factors.

\section{Declarations}

\section{Compliance with Ethical Standards}

All applicable international, national, and/or institutional guidelines for the care and use of animals were followed. This study was confirmed in ethic committee of Hamadan university of medical sciences (Code No: IR.UMSHA.REC.1398.1077).

All procedures were in accordance with the ethical standards of the institutional and/or national research committee and with the 1964 Helsinki declaration and its later amendments or comparable ethical standards. Informed consent: MSCs were isolated from human umbilical cord samples after filling out the consent form by parents.

\section{Acknowledgement}

This work was supported by Hamadan university of medical sciences financially (No: 990202405). Special thanks to high institute for research and education in transfusion medicine, Tehran, Iran for technical supports.

\section{Funding}

This work was supported by Hamadan university of medical sciences financially (No: 990202405).

\section{Conflict of interest}

The authors declare that they have no conflict of interest.

\section{Author contributions}

Conceptualization: [Fatemeh Amiri], Methodology: [ Sedigheh Molaei, Shirin Ferdowsi and Marzie Bahadori], Formal analysis: [Rasoul Salimi], Investigation and Writing - original draft preparation: [Sedigheh Molaie and Marzie Bahadori], Review and editing: [Fatemeh Amiri, Marzie Bahadori and Shirin Ferdowsi], Funding acquisition: [Fatemeh Amiri, Rasoul Salimi], Supervision: [ Fatemeh Amiri]. 


\section{References}

1. Kim WR, Brown RS Jr, Terrault NA, El-Serag H (2002) Burden of liver disease in the United States: summary of a workshop. Hepatology 36(1):227-242. https://doi.org/10.1053\%2Fjhep.2002.34734

2. Asrani SK, Devarbhavi H, Eaton J, Kamath PS (2019) Burden of liver diseases in the world. J Hepatol 70(1):151-171. https://doi.org/10.1016/j.jhep.2018.09.014

3. Pimpin L, Cortez-Pinto H, Negro F, Corbould E, Lazarus JV, Webber L et al (2018) Burden of liver disease in Europe: epidemiology and analysis of risk factors to identify prevention policies. $\mathrm{J}$ Hepatol 69(3):718-735. https://doi.org/10.1016/j.jhep.2018.05.011

4. Corey KE, Kaplan LM (2014) Obesity and liver disease: the epidemic of the twenty-first century. Clin Liver Dis 18(1):1-18. https://doi.org/10.1016/j.cld.2013.09.019

5. Pereira K, Salsamendi J, Casillas J (2015) The global nonalcoholic fatty liver disease epidemic: what a radiologist needs to know. J Clin Imag Sci 5. https://doi.org/10.4103/2156-7514.157860

6. Jin S-Z, Liu B-R, Xu J, Gao F-L, Hu Z-J, Wang X-H et al (2012) Ex vivo-expanded bone marrow stem cells home to the liver and ameliorate functional recovery in a mouse model of acute hepatic injury. Hepatobiliary Pancreat Dis Int 11(1):66-73. https://doi.org/10.1016/S1499-3872(11)60127-6

7. Strom SC, Cai H, Ellis E, Mitamura K, Miki T (2006) Bigger may not be better when it comes to hepatocytes. Liver Transpl 12(1):16-18. https://doi.org/10.1002/It.20593

8. Toniutto P, Bitetto D, Fornasiere E, Fumolo E (2019) Challenges and future developments in liver transplantation. Minerva Gastroenterol Dietol 65(2):136-152. https://doi.org/10.23736/S1121421X.18.02529-1

9. Stickel F, Schuppan D (2007) Herbal medicine in the treatment of liver diseases. Dig Liver Dis 39(4):293-304. https://doi.org/10.1016/j.dld.2006.11.004

10. Zhu SY, Jiang N, Jie T, Jing Y, Yue Z (2017) Antioxidant and Anti-aging Activities of Silybum Marianum Protein Hydrolysate in Mice Treated with D-galactose. Biomed Environmental Sci 30(9):623-631. https://doi.org/10.3967/bes2017.083

11. Abenavoli L, Izzo AA, Milić N, Cicala C, Santini A, Capasso R (2018) Milk thistle (Silybum marianum): A concise overview on its chemistry, pharmacological, and nutraceutical uses in liver diseases. Phytother Res 32(11):2202-2213. https://doi.org/10.1002/ptr.6171

12. Flora K, Hahn M, Rosen H, Benner K (1998) Milk thistle (Silybum marianum) for the therapy of liver disease. Am J Gastroenterol 93(2):139-143. https://doi.org/10.1111/j.15720241.1998.00139.x

13. Federico A, Dallio M, Loguercio C (2017) Silymarin/silybin and chronic liver disease: a marriage of many years. Molecules 22(2):191. https://doi.org/10.3390/molecules22020191

14. Bijak M (2017) Silybin, a major bioactive component of milk thistle (Silybum marianum L. Gaernt.)Chemistry, bioavailability, and metabolism. Molecules 22(11):1942.

https://doi.org/10.3390/molecules22111942

Page 13/21 
15. Papackova Z, Heczkova M, Dankova H, Sticova E, Lodererova A, Bartonova L et al (2018) Silymarin prevents acetaminophen-induced hepatotoxicity in mice. PLoS ONE 13(1):e0191353. https://doi.org/10.1371/journal.pone.0191353

16. Feher J, Lengyel G (2012) Silymarin in the prevention and treatment of liver diseases and primary liver cancer. Curr Pharm Biotechnol 13(1):210-217. https://doi.org/10.2174/138920112798868818

17. Ghaneialvar H, Soltani L, Rahmani HR, Lotfi AS, Soleimani M (2018) Characterization and classification of mesenchymal stem cells in several species using surface markers for cell therapy purposes. Ind J Clin Biochem 33(1):46-52. https://doi.org/10.1007/s12291-017-0641-x

18. Rodini CO, da Silva PBG, Assoni AF, Carvalho VM, Okamoto OK (2018) Mesenchymal stem cells enhance tumorigenic properties of human glioblastoma through independent cell-cell communication mechanisms. Oncotarget 9(37):24766. https://doi.org/10.18632/oncotarget.25346

19. Friedman SL, Arthur M (1989) Activation of cultured rat hepatic lipocytes by Kupffer cell conditioned medium. Direct enhancement of matrix synthesis and stimulation of cell proliferation via induction of platelet-derived growth factor receptors. J Clin Inves 84(6):1780-1785. https://doi.org/10.1172/JCl114362

20. Driscoll J, Patel T (2019) The mesenchymal stem cell secretome as an acellular regenerative therapy for liver disease. J Gastroenterol 5:1-11. https://doi.org/10.1007/s00535-019-01599-1

21. Qiu G, Zheng G, Ge M, Wang J, Huang R, Shu Q et al (2018) Mesenchymal stem cell-derived extracellular vesicles affect disease outcomes via transfer of microRNAs. Stem Cell Res Ther 9(1):320. https://doi.org/10.1186/s13287-018-1069-9

22. Tokhanbigli S, Baghaei K, Asadirad A, Hashemi SM, Asadzadeh-Aghdaei H, Zali MR (2019) Immunoregulatory impact of human mesenchymal-conditioned media and mesenchymal derived exosomes on monocytes. Mol Biol Res Commun 3:79-89. https://doi.org/10.22099/mbrc.2019.33346.1397

23. Sagaradze G, Grigorieva O, Nimiritsky P, Basalova N, Kalinina N, Akopyan Z et al (2019) Conditioned Medium from Human Mesenchymal Stromal Cells: Towards the Clinical Translation. Int J Mol Sci 20(7):1656. https://doi.org/10.3390/ijms20071656

24. Mannello F, Tonti GA (2007) Concise review: no breakthroughs for human mesenchymal and embryonic stem cell culture: conditioned medium, feeder layer, or feeder-free; medium with fetal calf serum, human serum, or enriched plasma; serum-free, serum replacement nonconditioned medium, or ad hoc formula? All that glitters is not gold! Stem Cell 25(7):1603-1609. https://doi.org/10.1634/stemcells.2007-0127

25. Wang Y-H, Wu D-B, Chen B, Chen E-Q, Tang H (2018) Progress in mesenchymal stem cell-based therapy for acute liver failure. Stem Cell Res Ther 9(1):227. https://doi.org/10.1186/s13287-0180972-4

26. Vizoso FJ, Eiro N, Cid S, Schneider J, Perez-Fernandez R (2017) Mesenchymal stem cell secretome: toward cell-free therapeutic strategies in regenerative medicine. Int J Mol Sci 18(9):1852. https://doi.org/10.3390/ijms18091852 
27. Amiri F, Halabian R, Dehgan Harati M, Bahadori M, Mehdipour A, Mohammadi Roushandeh A et al (2015) Positive selection of Wharton's jelly-derived CD105+ cells by MACS technique and their subsequent cultivation under suspension culture condition: A simple, versatile culturing method to enhance the multipotentiality of mesenchymal stem cells. Hematology 20(4):208-216. https://doi.org/10.1179/1607845414Y.0000000185

28. Amiri F, Molaei S, Bahadori M, Nasiri F, Deyhim MR, Jalili MA (2016) Autophagy-modulated human bone marrow-derived mesenchymal stem cells accelerate liver restoration in mouse models of acute liver failure. Iran Biomed J 20(3):135. https://doi.org/10.7508/ibj.2016.03.002

29. Zare H, Jamshidi S, Piryaei A, Dehghan MM, Sasani F, Molaei S et al (2015) Induction of acute hepatic failure by carbon tetrachloride in the nmri mouse model. Qom Univer Med Sci J 9(4):74-84

30. Ravan AP, Goudarzi F, Rafieemehr H, Bahmani M, Rad F, Jafari M et al (2019) Human umbilical cordmesenchymal stem cells conditioned medium attenuates $\mathrm{CCl} 4$ induced chronic liver fibrosis. Toxin Reviews 8:1-12. https://doi.org/10.1080/15569543.2019.1590849

31. Pawitan JA (2014) Prospect of stem cell conditioned medium in regenerative medicine. BioMed Res Int 2014. https://doi.org/10.1155/2014/965849

32. McGill MR, Cao M, Svetlov A, Sharpe MR, Williams CD, Curry SC et al (2014) Argininosuccinate synthetase as a plasma biomarker of liver injury after acetaminophen overdose in rodents and humans. Biomarkers 19(3):222-230. https://doi.org/10.3109/1354750X.2014.897757

33. Loosen SH, Schueller F, Trautwein C, Roy S, Roderburg C (2017) Role of circulating microRNAs in liver diseases. World J Hepatol 9(12):586. https://doi.org/10.4254/wjh.v9.i12.586

34. Gerlach CV, Vaidya VS (2017) MicroRNAs in injury and repair. Arch Toxicol 91(8):2781-2797. https://doi.org/ 10.1007/s00204-017-1974-1

35. Hermenean A, Stan M, Ardelean A, Pilat L, Mihali CV, Popescu C et al (2015) Antioxidant and hepatoprotective activity of milk thistle (Silybum marianum L. Gaertn.) seed oil. Open life sci 10(1). https://doi.org/10.1515/biol-2015-0017

36. Parekkadan B, Van Poll D, Suganuma K, Carter EA, Berthiaume F, Tilles AW et al (2007) Mesenchymal stem cell-derived molecules reverse fulminant hepatic failure. PLoS ONE 2(9):e941. https://doi.org/10.1371/journal.pone.0000941

37. Eguchi A, Wree A, Feldstein AE (2014) Biomarkers of liver cell death. J Hepatol 60(5):1063-1074. https://doi.org/10.1016/j.jhep.2013.12.026

38. Saeed WK, Jun DW (2014) Necroptosis: an emerging type of cell death in liver diseases. World J Gastroenterol 20(35):12526. https://doi.org/10.3748/wjg.v20.i35.12526

39. Zhang Y, Qiao HX, Zhou YT, Hong L, Chen JH (2018) Fibrinogen-like-protein 1 promotes the invasion and metastasis of gastric cancer and is associated with poor prognosis. Mol Med Rep 18(2):1465-1472. https://doi.org/10.3892/mmr.2018.9097

40. Xagorari A, Siotou E, Yiangou M, Tsolaki E, Bougiouklis D, Sakkas L et al (2013) Protective effect of mesenchymal stem cell-conditioned medium on hepatic cell apoptosis after acute liver injury. Int $\mathrm{J}$ Clin Exp Pathol 6(5):831 
41. Hu C, Zhao L, Duan J, Li L (2019) Strategies to improve the efficiency of mesenchymal stem cell transplantation for reversal of liver fibrosis. J Cell Mol Med 23(3):1657-1670. https://doi.org/ $10.1111 / \mathrm{jcmm} .14115$

42. Bi Z, Zhou Q, Geng Y, Zhang H (2016) Human umbilical cord mesenchymal stem cells ameliorate experimental cirrhosis through activation of keratinocyte growth factor by suppressing microRNA199. Eur Rev Med Pharmacol Sci 20(23):4905-4912. https://doi.org/ 10.1155/2021/6685605

43. Qavami N, Naghdi Badi H, Labbafi M, Mehrafarin A (2013) A Review on Pharmacological, Cultivation and Biotechnology Aspects of Milk Thistle (Silybum marianum (L.) Gaertn.). J Med Plants 3(47):1937

44. Koçarslan A, Koçarslan S, Aydin MS, Gunay Ş, Karahan MA, Taşkın A et al (2016) Intraperitoneal administration of silymarin protects end organs from multivisceral ischemia/reperfusion injury in a rat model. Braz J Cardiovas Surg 31(6):434-439. https://doi.org/10.5935/1678-9741.20160072

45. Lotfinia M, Kadivar M, Piryaei A, Pournasr B, Sardari S, Sodeifi N et al (2016) Effect of secreted molecules of human embryonic stem cell-derived mesenchymal stem cells on acute hepatic failure model. Stem Cell Dev 25(24):1898-1908. https://doi.org/10.1089/scd.2016.0244

46. An SY, Jang YJ, Lim H-J, Han J, Lee J, Lee G et al (2017) Milk fat globule-EGF factor 8, secreted by mesenchymal stem cells, protects against liver fibrosis in mice. Gastroenterology 152(5):1174-1186. https://doi.org/10.1053/j.gastro.2016.12.003

47. Huang B, Cheng X, Wang H, Huang W, Wang D, Zhang K et al (2016) Mesenchymal stem cells and their secreted molecules predominantly ameliorate fulminant hepatic failure and chronic liver fibrosis in mice respectively. J Transl Med 14(1):45. https://doi.org/10.1186/s12967-016-0792-1

48. Šuk J, Jašprová J, Biedermann D, Petrásková L, Valentová K, Křen V et al (2019) Isolated Silymarin Flavonoids Increase Systemic and Hepatic Bilirubin Concentrations and Lower Lipoperoxidation in Mice. Oxid Med Cell Longev 2019. https://doi.org/10.1155/2019/6026902

\section{Figures}



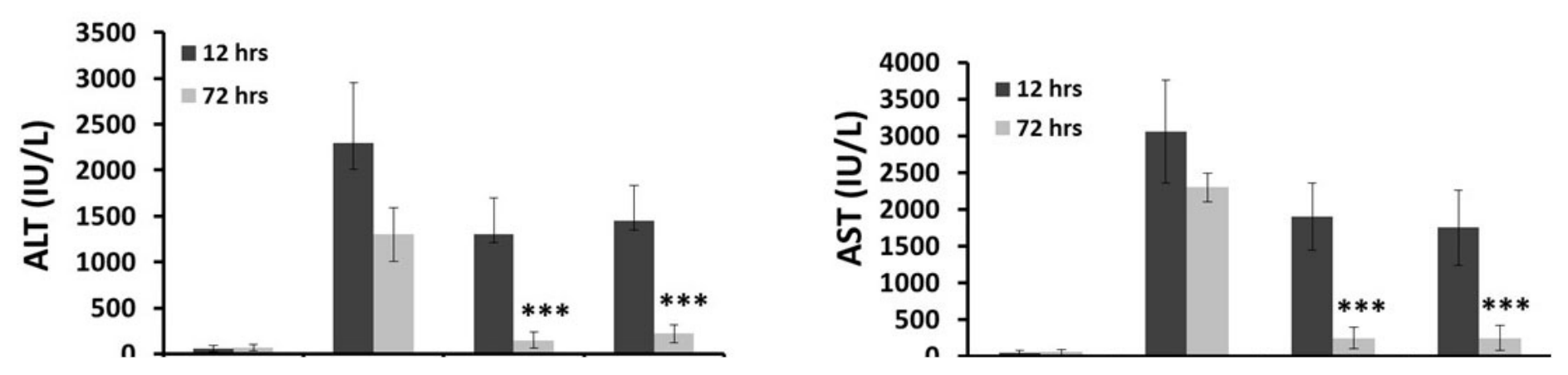

\section{Figure 1}

Evaluation of Silymarin or Sph-MSC-CM effects on liver enzymes and MicroRNA122 expression 12 and 72 hours after treatment. a. ALT levels. b. AST level. c. ASS concentration. ASS reduction was significantly detected in experimental groups (LF-SM and LF-MSC-CM) 12 hours after Silymarin or MSC$\mathrm{CM}$ administration. d. MicroRNA-122 fold change ratio related to $\beta$-actin in different groups 12 and 72 hours after treatment. Early decrease in the level of MicroRNA-122 was observed only in LF-MSC-CM 12 hours after Sph-MSC-CM administration. Date was shown as mean \pm SD. ${ }^{\star} p<0.05,{ }^{\star \star \star} * p<0.001$ LF-SM and LF-MSC-CM vs sham. AST: Aspartate aminotransferase, ALT: Alanine aminotransferase, ASS:

Argininosuccinate synthetase, Sph-MSC-CM: Conditioned medium from spheroid form of mesenchymal stem cell, LF-SM: LF mice receiving Silymarin, LF-MSC-CM: LF mice receiving Sph-MSC-CM. 

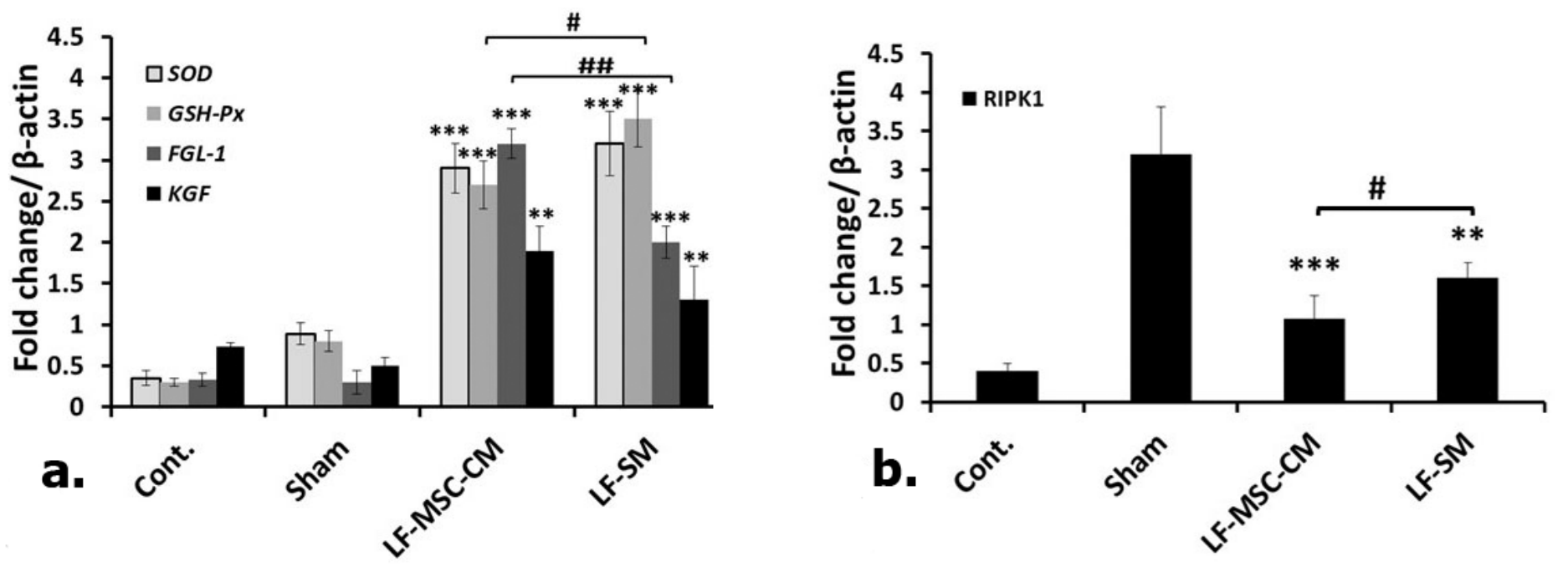

Figure 2

SOD, GSH-PX, KGF, FGL-1 and RIPK1 expression 72 hours after treatment. Real time-PCR was performed to quantified gene expression in liver homogenates. a. Gene expression level of SOD, GSH-PX, FGL-1 and KGF 72 hours after Silymarin or Sph-MSC-CM injection. Date indicated that SOD, GSH-PX, FGL-1 and KGF genes were upregulated in LF-SM and LF-MSC-CM in comparison with sham. Less expression of GSH-PX was detected in LF-MSC-CM compared with LF-SM. Moreover, LF-MSC-CM showed higher FGL-1 gene expression vs LF-SM. b. RIPK1 gene expression. Both LF-SM and LF-MSC-CM groups expressed downregulated level of RIPK1. The significantly lower RIPK1 expression was detected in LF-MSC-CM. All fold changes of gene expression were normalized by $\beta$-actin gene expression. Data was shown as mean \pm SD. ${ }^{* \star} p<0.01,{ }^{* \star *} p<0.001$ LF-SM and LF-MSC-CM vs sham. \# $p<0.05$, \#\# $p<0.01$ LF-MSC-CM $v s$ LF-SM. GSH-PX: Glutathione peroxidase, SOD: Superoxide dismutase, RIPK1: Receptor interacting protein kinases, FGL-1: Fibrinogen-like protein 1 and KGF: keratinocyte growth factor. Sph-MSC-CM: Conditioned medium from spheroid form of mesenchymal stem cell, LF-SM: LF mice receiving Silymarin, LF-MSC-CM: LF mice receiving Sph-MSC-CM. 

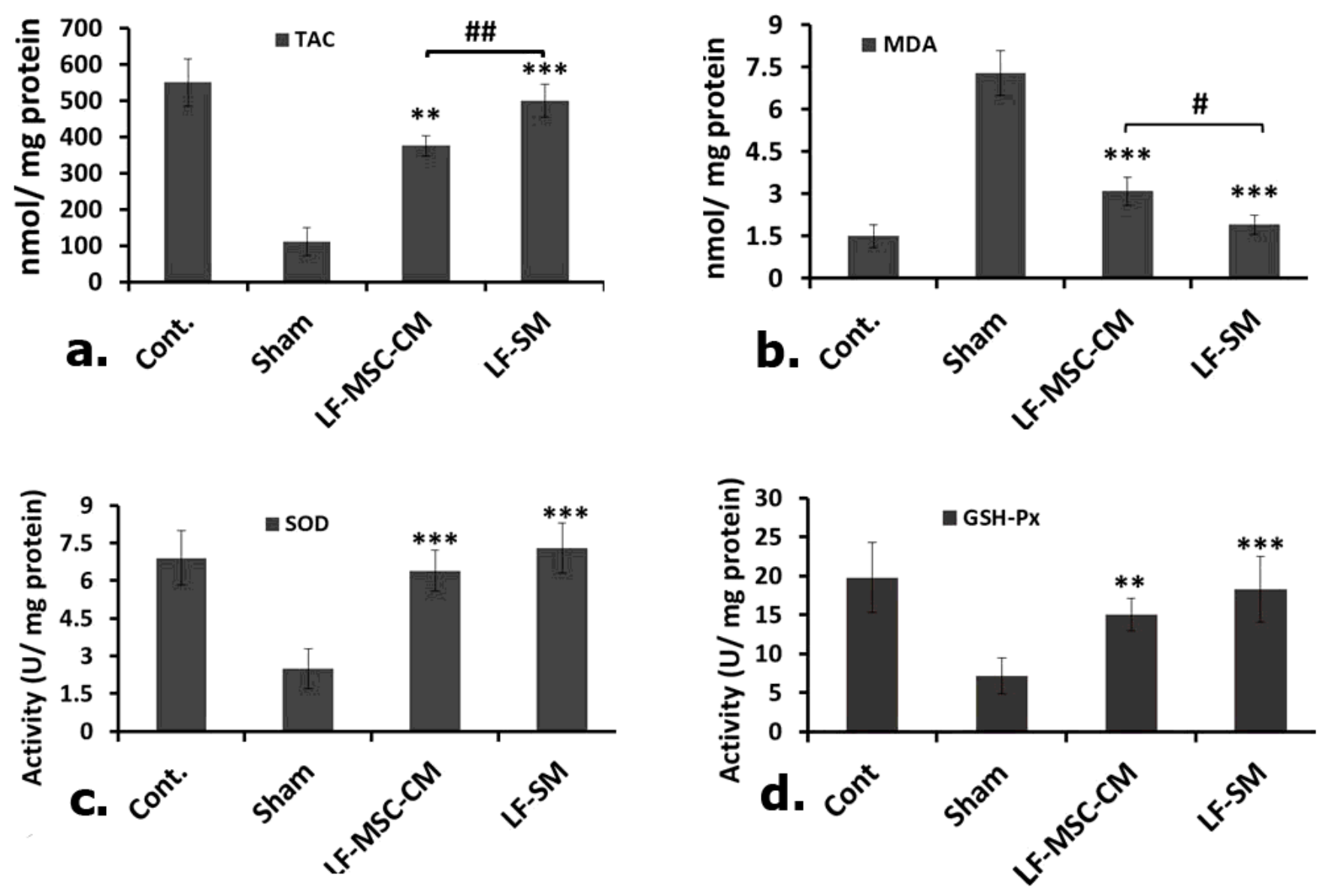

Figure 3

Analysis of oxidative stress status. MDA and TAC level as well as SOD and GSH-Px activity were determined in liver homogenates 72 hours after treatment. a. TAC level of different groups. TAC level was enhanced in both LF-SM and LF-MSC-CM when compared to sham. Significantly lower level of TAC was detected in LF-MSC-CM. b. MDA measurement in different groups. MDA amount was higher in LF-MSCCM. c. SOD activity of different groups. LF-MSC-CM and LF-SM had more SOD activity compared to sham. d. GSH-Px activity of different groups. High activity of GSH-Px was determined in both LF-MSC-CM and LF-SM groups with compared to sham. Date was shown as mean \pm SD. ${ }^{* \star} p<0.01,{ }^{* \star} p<0.001 \mathrm{LF}-\mathrm{SM}$ and LF-MSC-CM vs sham. \# $p<0.05$, \#\# $p<0.01$ LF-MSC-CM vs LF-SM. TAC: Total antioxidant capacity, MDA: Malondialdehyde, GSH-Px: Glutathione peroxidase, SOD: Superoxide dismutase, LF-SM: LF mice receiving Silymarin, LF-MSC-CM: LF mice receiving Sph-MSC-CM. 
Sham LF-SM LF-MSC-CM

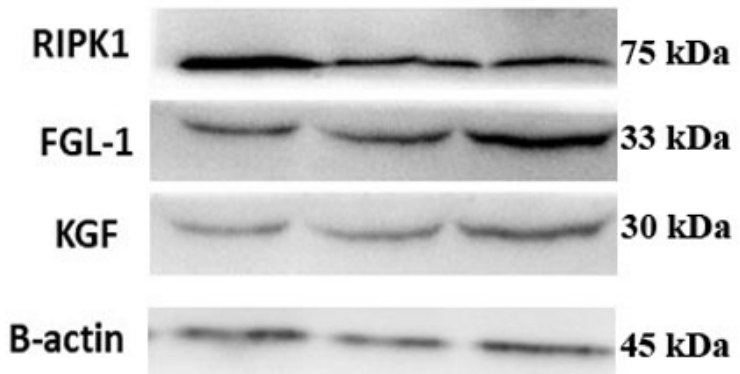

a.

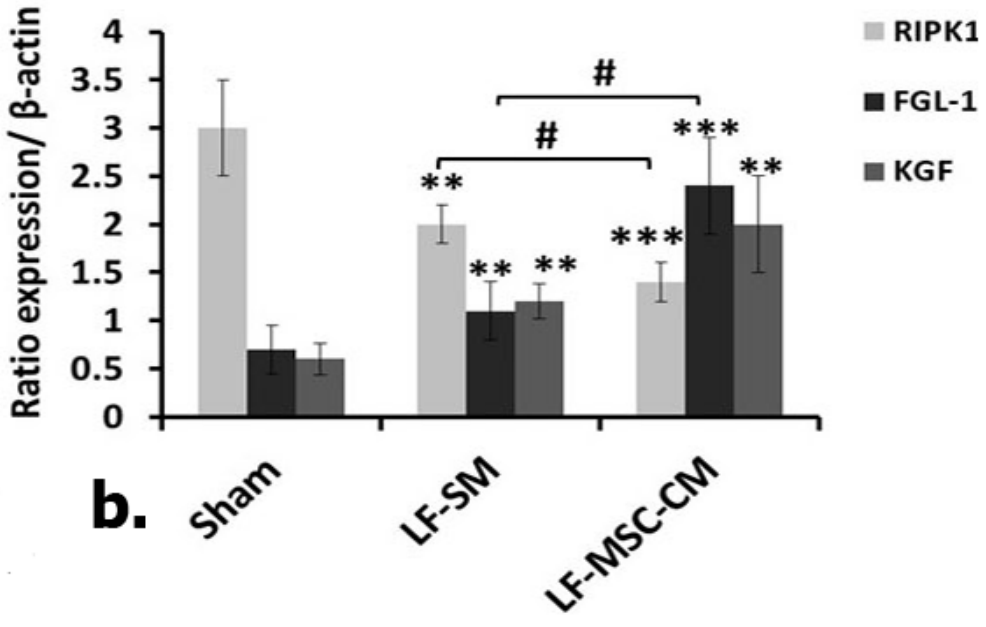

Figure 4

RIPK1, FGL-1 and KGF protein expression. Western blot analysis was performed to detect RIPK1, FGL-1 and KGF proteins in liver homogenates 72 hours after treatment. a. Expression of RIPK1, FGL-1 and KGF proteins in LF-SM and LF-MSC-CM. $\beta$-actin was used to normalize protein content. $\mathbf{b}$. Semi-quantification of western blot results. Both LF-MSC-CM and LF-SM not only expressed more FGL-1 and KGF proteins but also produced less RIPK1 protein. Significantly more amount of FGL-1 was determined in LF-MSC-CM. Moreover, LF-MSC-CM expressed lower level of RIPK1 against LF-SM. Date was shown as mean \pm SD. ${ }^{* \star} p<0.01,{ }^{* \star \star} p<0.001$ LF-SM and LF-MSC-CM $v s$ sham. \# $p<0.05$ LF-MSC-CM $v s$ LF-SM. RIPK1: Receptor interacting protein kinases, FGL-1: Fibrinogen-like protein 1 and KGF: keratinocyte growth factor. LF-SM: LF mice receiving Silymarin, LF-MSC-CM: LF mice receiving Sph-MSC-CM.

\section{Figure 5}

Histopathological analysis of liver sections and mice survival rate. Liver sections of different groups were prepared and stained with hematoxylin and eosin (H\& E) 72 hours after treatment. a. Intralobular necrotic bridges along with several necrotic and inflammatory cells were observed in sham. No intralobular necrotic bridges were seen in LF-MSC-CM and LF-SM groups. b. Quantification of liver necrosis and inflammation. The numbers of intralobular necrotic bridge, necrotic cells and inflammatory cells were estimated with high-power field (HPF) light microscopy at least in 30 fields. Less necrotic and inflammatory cells along with no interalobular necrotic bridges in liver sections of LF-MSC-CM indicated better necrosis control in this group. c. The 14-days survival rate of different animal groups. More mean of survival time was recorded for LF-MSC-CM. Date was shown as mean \pm SD. ${ }^{* \star} p<0.001$ LF-SM and LF-

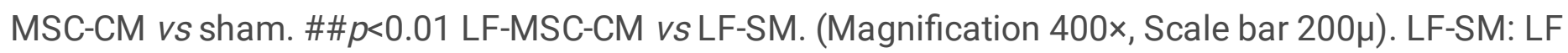
mice receiving Silymarin, LF-MSC-CM: LF mice receiving Sph-MSC-CM. 


\section{Supplementary Files}

This is a list of supplementary files associated with this preprint. Click to download.

- Suplementaryinformation.docx 